\title{
Correction to: The development of a healing model of care for an Indigenous drug and alcohol residential rehabilitation service: a community-based participatory research approach
}

\author{
Alice Munro ${ }^{1 *}$, Anthony Shakeshaft ${ }^{1}$ and Anton Clifford ${ }^{1,2}$
}

\section{Correction}

Upon publication of the original article (Munro et al., 2017), the authors noticed the following errors:

1. A few words are missing in the conclusion of the Abstract. It needs to read:

Conclusion: The description of the CBPR process and the Healing Model of Care provides one possible solution about how to provide better care for the large and growing population of Indigenous people with substance misuse issues

2. On page 4, at the top of the page: "Step 1: Effective engagement..." should be "Step 1: Initial engagement..."

3. On page 5, in the middle of first column, the start of the sentence "The semi-structured interviews used 'yarning' approach" should be: "The semi-structured interviews used a research "yarning' approach"

4. On page 6 , in the section with the sub-heading "Healing through culture and country", "red centre of circle" should be: "in the centre circle"

5. Table

a) The "Aftercare" core treatment component at the bottom of column $b$ is missing

b) "b. Intervention" should instead read "b. Treatment" Please see the corrected Table 1 below.
6. The second sentence of the Discussion should be: "The Healing Model of Care proposed in this paper is based on the premise that successful treatment in a remote Indigenous drug and alcohol residential rehabilitation service will improve clients' quality of life and cultural connectedness which will, in turn, be strongly associated with sustained reductions in their risky substance use."

7. On page 10, the following sentence requires two corrections: "We recognise other outcome measures, namely the World Health Organization Quality of Life - BREF (abbreviated version; WHOQoL-BREF) is not currently validated for use with Indigenous peoples, but given that health education and behaviour studies are tested for validity and reliability inconsistently (Berry et al., 2013) and there have been no measures designed and validated for use within Indigenous drug and alcohol residential rehabilitation settings, the authors consider this a pivotal area for future research (Stephens et al., 2013; James et al., 2017, under review)."

a) the first mention of "is" should be "are"

b) The reference "Barry et al., 2013" should be "Berry et al. 2013"

8. In the References section, the word "Islander" in the term "Aboriginal and Torres Strait Islander" also should be capitalised. The following references in the reference list need this change to be made:

AIHW, 2017;

DOHA, 2013;

Doyle et al., 2015

${ }^{1}$ National Drug and Alcohol Research Centre, University of New South Wales, Sydney, NSW 2052, Australia

Full list of author information is available at the end of the article 


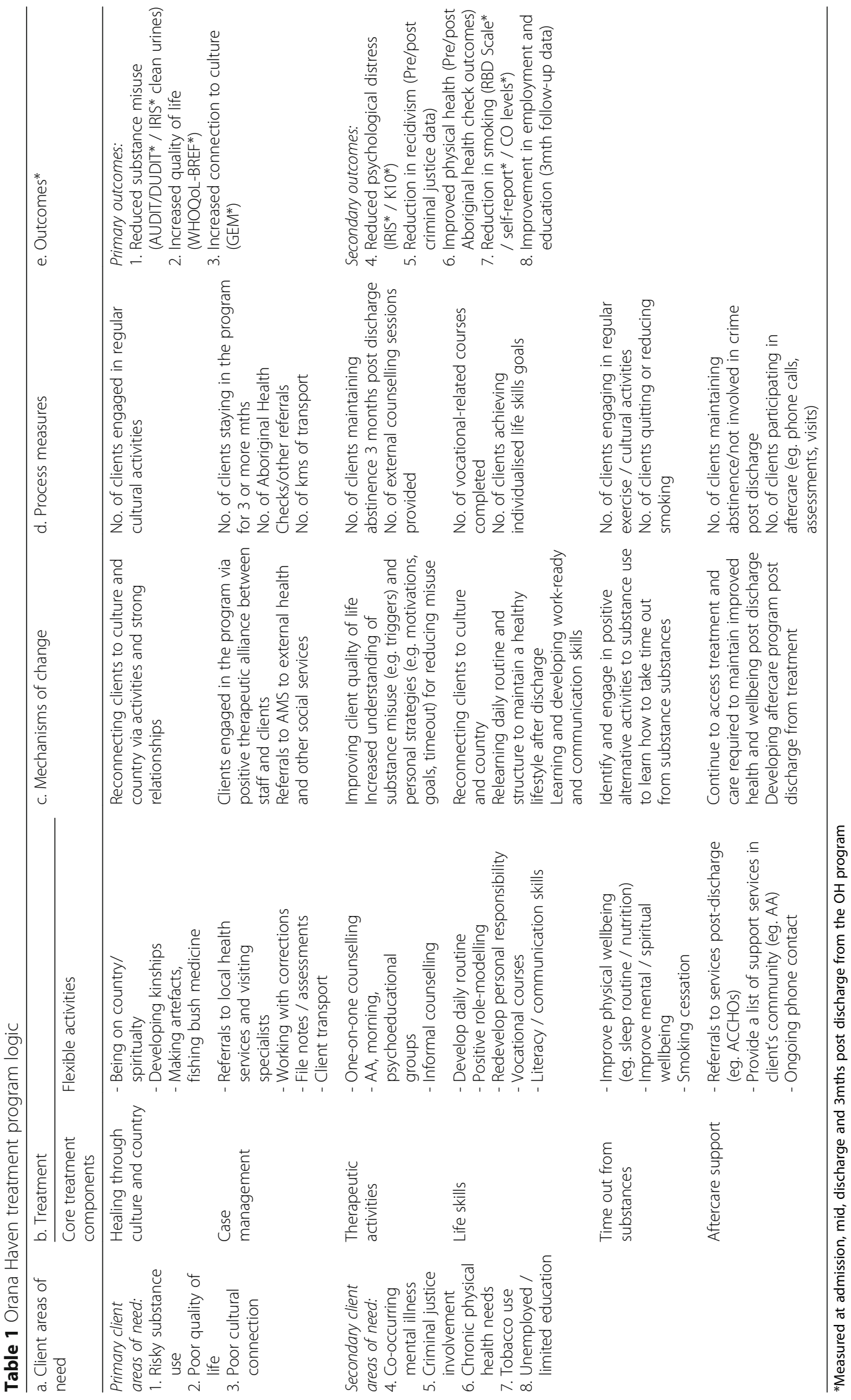


Doyle et al., This also needs a capital "N" in "NSW"

Gould et al., 2014;

Heffernan et al., 2016;

NH\&MRC, 2013;

NIDAC, 2014;

QSA, 2008.

Marmot, 2011. This also needs a capital "I" in

"Indigenous"

\section{Author details}

${ }^{1}$ National Drug and Alcohol Research Centre, University of New South Wales, Sydney, NSW 2052, Australia. 'University of Queensland, Brisbane, QLD 4072, Australia.

Received: 21 January 2018 Accepted: 26 January 2018

Published online: 14 March 2018

\section{Reference}

Munro, A, Shakeshaft, A, Clifford, A. (2017). The development of a healing model of care for an Indigenous drug and alcohol residential rehabilitation service: a community-based participatory research approach. Health \& Justice, 5, 12. https://doi.org/10.1186/s40352-017-0056-Z. 